\section{The Mathematics and Biology of the Biodistribution of Radiopharmaceuticals: A Clinical Perspective}

\author{
W.C. Klingensmith III
}

New York, NY: Springer, 2016, 274 pages, $\$ 109$

Nuclear medicine relies on capturing the biodistribution of radiopharmaceuticals on images that can be interpreted by the physician. The Mathematics and Biology of the Biodistribution of Radiopharmaceuticals: A Clinical Perspective uses mathematic equations to describe the distribution of radiopharmaceuticals. The mathematics provide a conceptual basis for the biodistribution of the radiopharmaceutical. As the author points out so eloquently, the most comprehensive method to image interpretation in nuclear medicine includes "conceptual knowledge of the mathematic equations that describe the quantitative relationships among the various biologic parameters." Although, at times, the details of the mathematics are challenging to follow, the author does a good job at adding meticulous illustrations and explanations to guide readers to an understanding of the equations. By the end of the book, readers better understand that visual assessment of images in nuclear medicine can be further clarified and explained by numbers.

The book is divided into 3 parts, and its primary focus is to use mathematics to explain the biologic and physiologic processes that influence the biodistribution of radiopharmaceuticals depicted in the images. Part I provides a historical background to the field of nuclear medicine and discusses two ways to evaluate images: visual versus quantitative interpretation. Here, the author points out that quantitation can enhance interpretation of images and is most effective when used together with visual interpretation. Again, the author is effective in engaging readers with figures, images, and tables; but it should be noted that Table 2.3 contains an error, listing I-123 as !-123.

Part II dives into the mathematics that explain the biologic processes influencing biodistribution of the imaging agent. Although therapeutic procedures are not discussed in this book, it is stated that the concepts of the equations for diagnostic studies will also apply to corresponding therapeutic radiopharmaceuticals. The author presents elaborate equations to explain, conceptually, what is happening in the body with the drug that is being captured in the image. Each chapter introduces different equations that relate to nuclear medicine studies, such as clearance, central volume principal, first-circulation time-activity curves, convolution analysis, measurement of relative and absolute function, and lastly a section on other quantitative techniques. The equations involve biologic processes that are related to the time-dependent biodistribution of radiopharmaceuticals. When an equation is presented, the author breaks down each component of the equation and allows for a biologic description for each mathematic factor. This helps the reader to better understand that each biologic parameter affects the final outcome seen in the clinical images. Further, to emphasize relevance, at the end of these chapters the author provides examples of clinical applications for the equations.

After the book has given the readers an understanding of the mathematics of diagnostic agents, part III dives into the quantitative evaluation of 39 different nuclear medicine studies. For studies not included, the author offers explanations as to why they were omitted, with the predominate reason being that the studies are rarely performed. Overall, part III is the most pragmatic for readers, such as myself, who are not nuclear medicine clinicians and thus are primarily exposed to images only to explain disease. Chapters 12-20 cover each diagnostic study, and the discussion of the nuclear medicine study is divided into subsections to include an overview, radiopharmaceutical characteristics, extraction mechanism, extraction efficiency, interventions, imaging, protocol design, and quantitative measurements. Many of the 39 studies reviewed do not involve quantification of a functional parameter; however, the author notes that in these studies the biodistribution of the imaging agent can nonetheless be explained through mathematics.

This is a concise and comprehensive book to provide perhaps a different perspective in explaining the images produced in diagnostic nuclear medicine procedures. Because this book is intended for nuclear medicine physicians, the clinical content presented is, at times, difficult to follow for nonphysician readers. However, the author effectively incorporates figures, tables, images, and diagrams to further drive the point home. For those who are interested in exploring other approaches to nuclear medicine, The Mathematics and Biology of the Biodistribution of Radiopharmaceuticals: A Clinical Perspective is recommended.

Dao Le

M.D. Anderson Cancer Center 1515 Holcombe Blvd,

Houston, TX 77030

E-mail: dble@mdanderson.org

Published online Feb. 9, 2017.

DOI: $10.2967 /$ jnumed.117.191312 